\title{
Development of an external-to-internal convertible endoscopic biliary drainage device - a preliminary prospective feasibility study
}

\section{다 (우우}

\author{
Authors \\ Akihiro Mori, Shun Ito, Takayuki Yumura, Hiroki Hachiya, Masashi Sawada, Shintaro Hayashi, Noritsugu Ohashi
}

Institution

Department of Gastroenterology, Ichinomiya Nishi

Hospital, Aichi, Japan

submitted 17.8 .2017

accepted after revision 15.11 .2017

\section{Bibliography}

DOI https://doi.org/10.1055/s-0043-123934 |

Endoscopy International Open 2018; 06: E123-E126

(c) Georg Thieme Verlag KG Stuttgart · New York

ISSN 2364-3722

\section{Corresponding author}

Akihiro Mori, Ichinomiya Nishi Hospital - Gastroenterology, 1 Kaimei-hira Ichinomiya Aichi Japan, Ichinomiya 494-0001, Japan

Fax: +81-586-48-0038

a-mori@anzu.or.jp

\section{ABSTRACT}

Background and study aims Endoscopic nasobiliary drainage (ENBD) for a malignant stricture in the bile duct has some advantages over endoscopic biliary stenting (EBS). However, ENBD may cause nasopharyngeal discom- fort. We developed an external-to-internal convertible endoscopic biliary drainage (ETI-EBD) device that enables both internal and external drainage to occur during a single endoscopy.

Patients and methods This device consists of three parts, comprising a 5-Fr ENBD tube $(250 \mathrm{~cm})$ (ENBD-t), an $8.5-\mathrm{Fr}$ EBS tube $(7 \mathrm{~cm})(E B S-t)$, and an $8-\mathrm{Fr}$ pusher tube for EBS $(230 \mathrm{~cm})(\mathrm{P}-\mathrm{t})$. The EBS- $t$ is mounted over the ENBD-t at the distal end of the ENBD-t. The P-t is also placed over the ENBD-t. After an endoscopic sphincterotomy, the EBS-t of the device is inserted into the papilla, then the duodenal endoscope is withdrawn, leaving the device in place. After ENBD, only the ENBD-t was withdrawn from the P-t. At this point, the EBS-t was isolated and left without endoscopy or radiography.

Results ETI-EBD was successfully placed in all consecutive 21 patients (100\%). The release of EBS-t from ENBD-t wit was successfully completed in 19 patients (90.5\%). There were 4 patients with kink of P-t when exchanging this device from the mouth to the nose. It was difficult for 2 patients to withdraw the ENBD-t because of poor lubrication performance. There were no significant complications associated with the use of the device.

Conclusion This device allows for both external and internal biliary drainage with a single endoscopy.

\section{Introduction}

Endoscopic nasobiliary drainage (ENBD) for a malignant stricture in the bile duct has some advantages over endoscopic biliary stenting (EBS). ENBD, allowing external drainage, enables not only drainage but also histological and bacteriological examination. It also contributes to diagnosis by enabling detailed cholangiography after drainage. However, ENBD may cause stress due to naso-pharyngeal discomfort. EBS obviates this stress, but it makes the above-mentioned examinations almost impossible. Therefore, we have developed an external-to-internal convertible endoscopic biliary drainage (ETI-EBD) device that enables both internal and external drainage to occur dur- ing a single endoscopy. Herein, we report the method and the feasibility of this device.

\section{Patients and methods}

\section{ETI-EBD device}

The device consists of three parts, comprising a 5-Fr ENBD tube $(250 \mathrm{~cm})($ ENBD-t) ( Fig. 1a), an 8.5-Fr EBS tube $(7 \mathrm{~cm})$ (EBS-t) ( $\triangleright$ Fig. 1b), and an 8-Fr pusher tube for EBS $(230 \mathrm{~cm})$ (P-t) ( $\triangleright$ Fig.1c). The EBS-t and the P-t (PBD-V631P-0807]; Olympus, Tokyo, Japan) are mounted over the ENBD-t (Flexima Nasobiliary catheter 5Fr; Boston Scientific, Marlborough, MA, USA). The EBS-t is positioned $12 \mathrm{~cm}$ from the distal end of the ENBD-t, while the $\mathrm{P}-\mathrm{t}$ is placed $10 \mathrm{~cm}$ proximal to the end of 


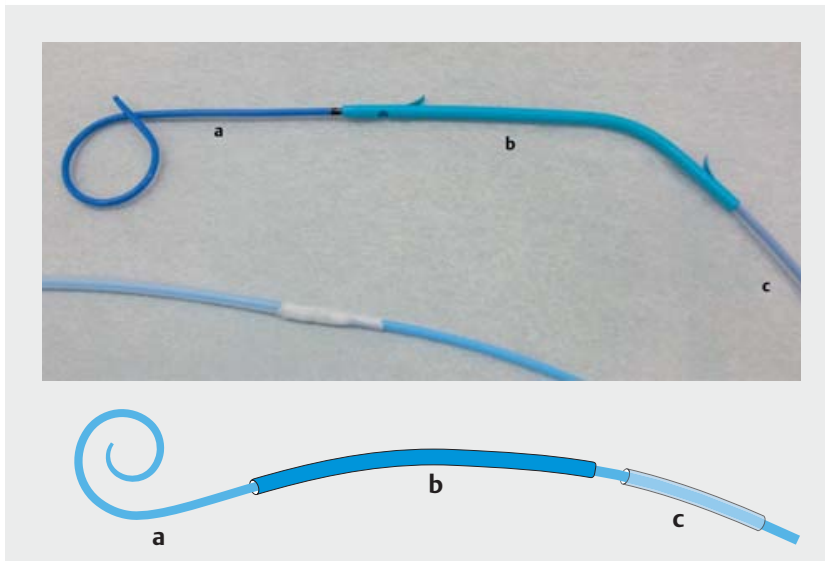

- Fig. 1 The device consists of 3 parts, comprising a 5-Fr ENBD tube $(250 \mathrm{~cm})($ ENBD-t) (a), an $8.5-\mathrm{Fr}$ EBS tube $(7 \mathrm{~cm})($ EBS - $\mathrm{t})(\mathrm{b})$, and an 8 -Fr pusher tube for EBS $(230 \mathrm{~cm})(\mathrm{P}-\mathrm{t})(\mathrm{c})$. The EBS-t and the P-t are mounted over the ENBD-t. The EBS- $t$ is positioned $12 \mathrm{~cm}$ from the distal end of the ENBD-t. The P-t is placed $10 \mathrm{~cm}$ proximal to the end of the ENBD-t. The proximal end of the P-t is firmly fastened to the ENBD-t.

the ENBD-t. The proximal end of the P-t is firmly fastened to the ENBD-t. Insertion of the ETI-EBD device is performed similar to that of a conventional ENBD-t. After passing the guidewire through the stricture in the bile duct via endoscopic retrograde cholangiopancreatography (ERCP), an endoscopic sphincterotomy was performed. Then the device was inserted over the guidewire through the endoscopic channel. Once the proximal end of the EBS-t was inserted into the papilla, the duodenal endoscope was withdrawn, leaving the device in place ( $\triangleright$ Fig. 2a). Then, the device was exchanged from the mouth to the transnasal route. At that time, both the EBS-t and P-t remained secured over the ENBD-t ( $>$ Fig.3a). After ENBD was performed, including detailed cholangiography, sufficient biliary drainage and bile collection, external-to-internal conversion was performed at the earliest possible date to minimize stress. The conversion was as follows: the fixation between the ENBD-t and P-t was released and only the ENBD-t was withdrawn from the P-t; the P-t was gripped so as not to be withdrawn with the ENBD-t under radiography. Then, the EBS-t was isolated from the P-t and ENBD-t ( $>$ Fig. 2b). Finally, we were able to leave only the EBS-t between the duodenum and the bile duct without endoscopy ( $\triangleright$ Fig. 2c and $\triangleright$ Fig. $3 \mathbf{b}$ ).

\section{Study design}

We evaluated the ETI-EBD device in a preliminary, prospective feasibility study. We enrolled 21 patients from 49 consecutive patients with a malignant biliary stricture between January 2015 and December 2016. Excluding criteria were a hilar part biliary stricture (17 patients), a history of esophagogastrointestinal surgery (one patient), an unsuccessful cannulation into the deep biliary tract ( 6 patients) and a disapprobative patient for this study (4 patients). The primary endpoint was to assess the feasibility and safety of placement of this device with the duodenal endoscopy. The secondary endpoint was to assess the feasibility and safety of release of EBS-t from ENBD-t without endoscopy. All patients provided written informed consent prior to participating in this study. This study was registered in a clinical trial database on January 30, 2015 (University Hospital Medical Information Network: UMIN000016388), and was approved by the hospital review board for human research.

\section{Results}

Twenty-one patients (mean age 79.8; range 69-90, male/female; 10/11, 19 distal part biliary strictures and two papillary tumors) were evaluated. The 22 patients excluded from this study underwent ENBD. Although cannulation into the common bile duct was unsuccessful in 6 patients (five distal part biliary strictures and one papillary tumor), they were all salvaged with the biliary drainage technique under endoscopic ultrasound. Placement of ETI-EBD device was successful in all 21 patients (100\%). Median placement time (from insertion of this device to fixation in the nose) was $4(2-17)$ minutes. There were no significant clinical complications associated with the placement but there were four patients with kink of P-t that occurred in the nasal cave when exchanging this device from the mouth to the nose. All patients with kink of P-t were repaired by hauling the kinked part out of the nose. Release of EBS-t from ENBD-t without endoscopy was successful in 19 patients (90.5\%). Although in 2 patients withdrawal of the ENBD-t was difficult because of poor lubrication, release was enabled by injection of lubrication into the space between P-t and ENBD-t. Median time of placement of the device before release of ENBD-t was 24 hours (range 1 -48). Median time of placement of the EBS-t after removal of ENBD-t was 31 days (range 7 117). Reasons for discontinuation of EBS were EBS-t obstruction in 13 patients (median patent time 27 days [range $7-70$ ]) including 8 patients who were converted to a metallic stent, performance of surgery for the stricture in 3 patients, and death in 5 patients. There were no significant complications associated with use of the device.

\section{Discussion}

There has been controversy regarding which drainage method, ENBD or EBS, is better for safety and efficacy. ENBD not only enables histological and bacteriological examination and detailed cholangiography after drainage [1], but also is superior in terms of reduction in rates $f$ preoperative cholangitis and postoperative pancreatic fistula [2]. However, ENBD, allowing external drainage, may interfere with reabsorption of bile and may induce bacterial translocation and interception of liver regeneration $[3,4]$. In that respect, ENBD is suitable for temporary placement but may be inadequate for prolonged drainage. In fact, when a patient in whom an ENBD was initially placed needs prolonged drainage, conversion to EBS is often performed with another endoscopic session. Thus, a method that could make conversion from ENBD to EBS easier, with a single endoscopy, would be useful. However, there have been few reports of such a method. Uchida et al. [5,6] reported on use of a conversion technique from external nasobiliary drainage to in- 

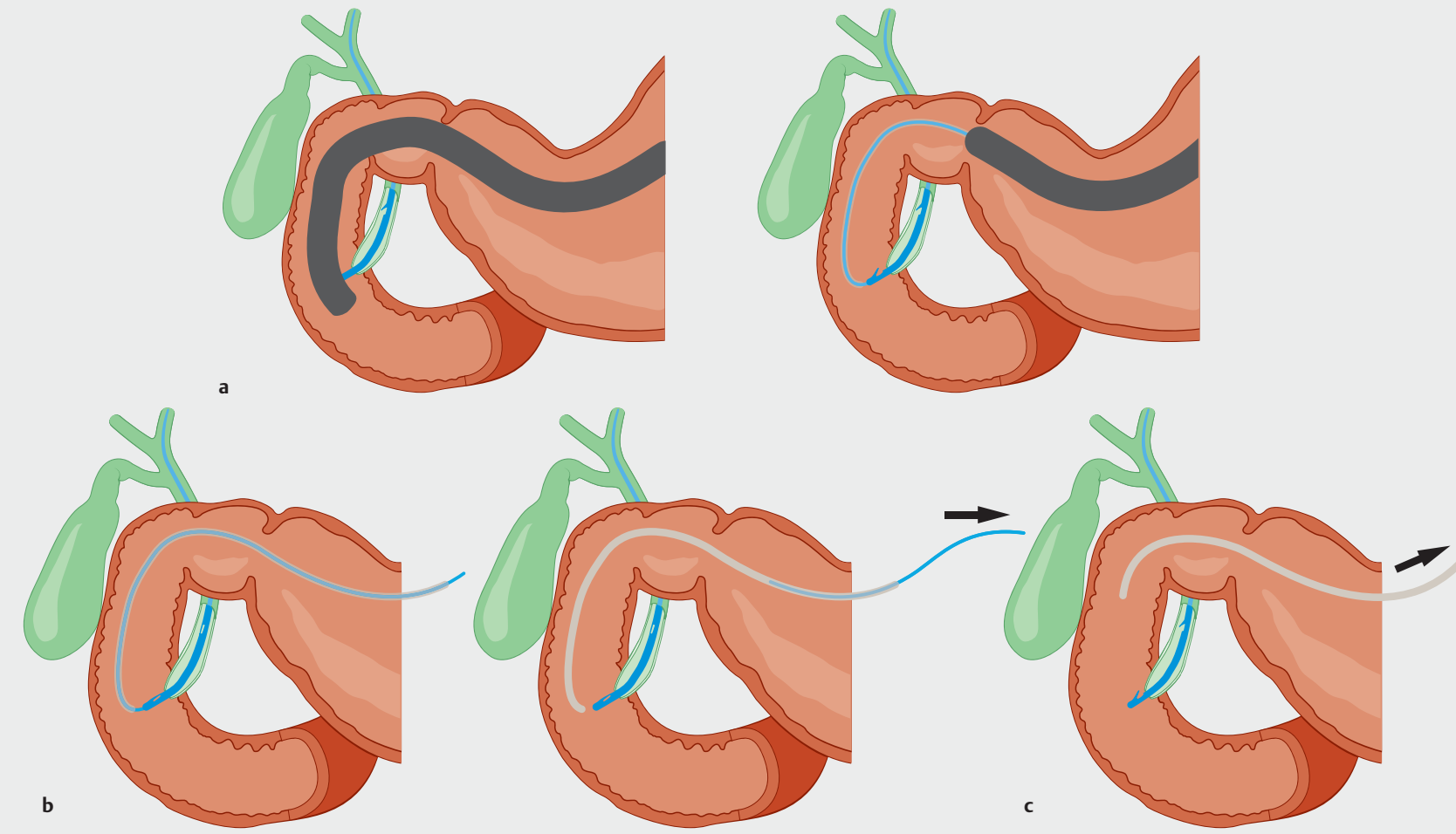

Fig. 2 a The device was inserted over the guidewire through the endoscopic channel. When the proximal end of the EBS-t was inserted to the papilla, the duodenal endoscope was withdrawn, leaving the device in place. $\mathbf{b}$ Fixation between the ENBD-t and the P-t was released and only the ENBD-t was withdrawn from the P-t. At this point, the P-t was gripped so as not to be withdrawn with the ENBD-t. Then, the EBS- $t$ was isolated from the P-t and ENBD-t. c We were able to leave only the EBS-t between the duodenum and the bile duct without endoscopy or radiography.
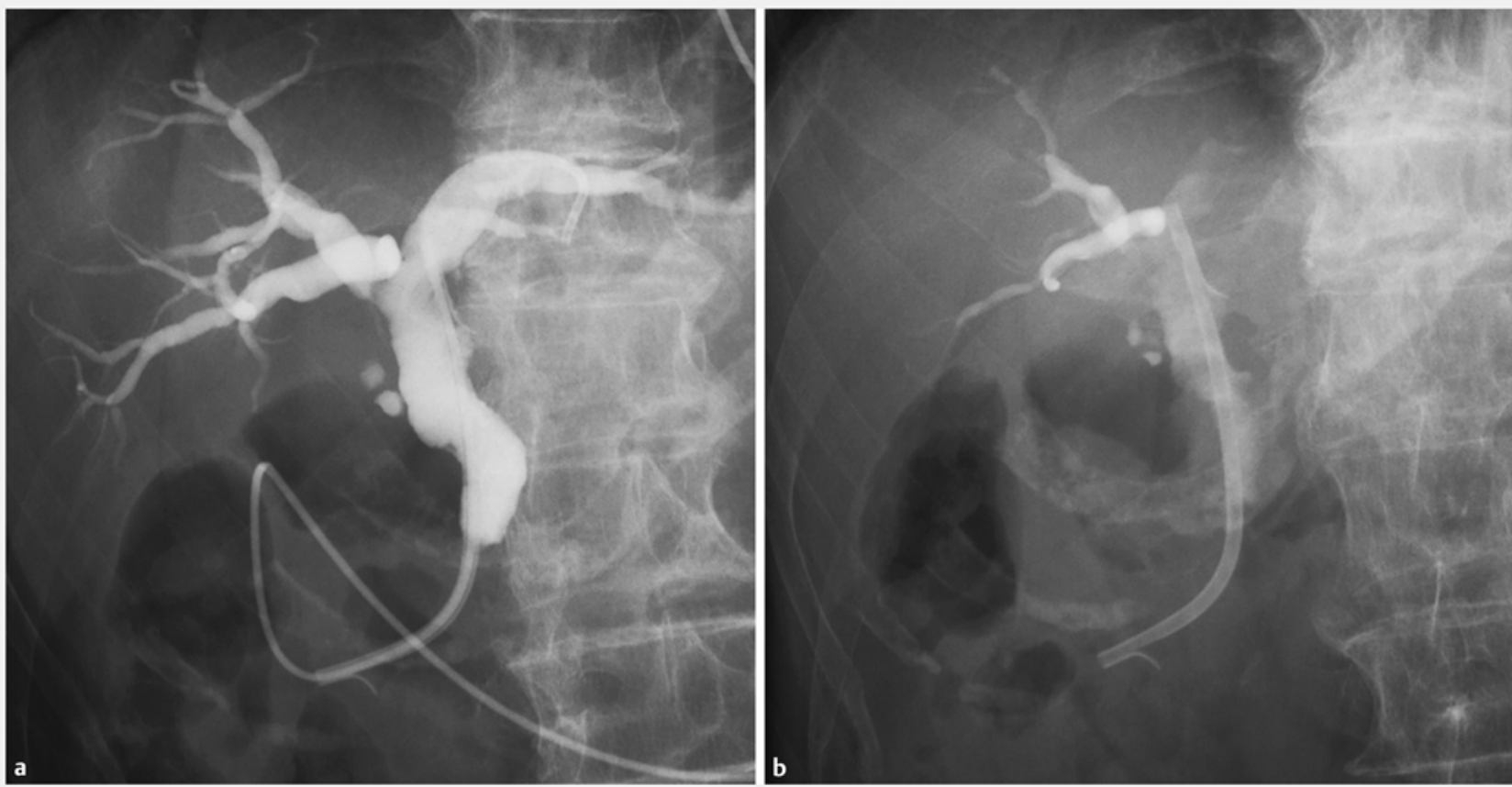

Fig. 3 a Both the EBS-t and P-t remained secured over the ENBD-t after the duodenal endoscope was withdrawn. $\mathbf{b}$ The EBS-t was isolated from P-t and the ENBD-t, leaving only the EBS-t between the duodenum and the bile duct. 
ternal drainage under endoscopy. That technique involved ENBD being cut with scissor forceps in the stomach under endoscopy. Although the technique did not require exchange of the ENBD tube, another endoscopic session was needed to cut the ENBD tube. Moreover, concern existed about potential drawbacks such as dislodgment and mucosal damage from the cut edge of the tube. In that regard, our method makes it possible to insert an external-to-internal convertible drainage device with a single endoscopy, and it was found to be safe for the digestive organ because all parts of the device are made of materials approved for human use. Because this study showed a high rate of success with use of the ETI-EBD device, we surmise that it may offer a useful medium for biliary tract drainage.

Although an EBS-t is bound to exchange to another EBS-t or EMS because of occlusion or infection associated with longterm placement, some patients in this study had relatively early occlusion. Because we performed external-to-internal conversion at the earliest possible date to minimize stress in principle, ENBD withdrawal may have been done before biliary drainage was sufficient to remove biliary debris.

There were some limitations of the usage of the ETI-EBD device. The device was cumbersome to manipulate as P-t was formed relatively easily from kink and drawing ENBD-t from P-t was sometimes hard because of poor lubrication. In addition, because the P-t was not designed as a catheter to be retained in the body, there may be a problem with its long-term use for ENBD. Currently, the ETI-EBD device seems to be acceptable for short-term usage. We believe that the best indication for this device is temporary placement before operation for a resectable malignant stricture. We do note that the ETI-EBD device seems to require further improvement and validation.

\section{Conclusion}

We have introduced a new device that has shown favorable feasibility and has enabled performance of both external and internal biliary drainage with a single endoscopy.

\section{Competing interests}

None

References

[1] Yagioka $\mathrm{H}$, Hirano $\mathrm{K}$, Isayama $\mathrm{H}$ et al. Clinical significance of bile cytology via an endoscopic nasobiliary drainage tube for pathological diagnosis of malignant biliary strictures. J Hepatobiliary Pancreat Sci 2011; 18: $211-215$

[2] Lin H, Li S, Liu X. The safety and efficacy of nasobiliary drainage versus biliary stenting in malignant biliary obstruction: A systematic review and meta-analysis. Medicine (Baltimore) 2016; 95: e5253

[3] Welsh FK, Ramsden CW, MacLennan K et al. Increased intestinal permeability and altered mucosal immunity in cholestatic jaundice. Ann Surg 1998; 227: 205-212

[4] Kamiya S, Nagino M, Kanazawa $\mathrm{H}$ et al. The value of bile replacement during external biliary drainage: an analysis of intestinal permeability, integrity, and microflora. Ann Surg 2004; 239: 510 - 517

[5] Uchida N, Ezaki T, Fukuma $\mathrm{H}$ et al. Conversion of endoscopic nasobiliary drainage to internal drainage by means of endoscopic scissor forceps. Endoscopy 2002; 34: 180

[6] Uchida N, Tsutsui H, Ezaki T et al. Conversion from external nasobiliary drainage to internal drainage using endoscopically available scissor forceps. Hepatogastroenterology 2003; 50: $1891-1893$ 